Reprod. Nutr. Dévelop., 1984, 24 (2), 137-145.

\title{
Action temporelle d'ecdystéroïdes sur la synthèse protéique ovarienne in vitro chez le Crustacé isopode terrestre Porcellio dilatatus (Brandt)
}

\author{
M. GOHAR, Catherine SOUTY
}

University de Poitiers, Laboratoire de Biologie Animale, "Physiologie et Génétique des Crustacés », E.R.A. CNRS No 230, 40, avenue du Recteur-Pineau, 86022 Poitiers Cedex, France.

Summary. The temporal action of ecdysteroids on ovarian protein synthesis in vitro in the terrestrial crustacean Isopoda, Porcellio dilatatus (Brandt).

In vitellogenic females of Porcellio dilatatus at stage $\mathrm{Cb}$ of the molt cycle $(15$ to 20 days after molting), ovarian protein synthesis could be stimulted in vitro by ecdysone and 20-hydroxyecdysone. These ecdysteroids used at subphysiological concentrations showed diurnal variations in their effects on ovarian protein synthesis. The diurnal rhythms of these effects of ecdysone were bimodal and those of 20-hydroxyecdysone unimodal.

\section{Introduction.}

L'intervention des ecdystéroïdes dans la formation du vitellus chez les femelles adultes d'insectes, où la glande de mue involue, est dans la plupart des cas douteuse ou controversée (Hoffmann, 1980 ; Hoffmann et al., 1980 ; Fuchs et Kang, 1981 ; Handler, 1982).

A l'inverse, chez les Crustacés, la majorité des auteurs accordent à ces hormones un rôle dans la vitellogenèse à travers les relations mue-vitellogenèse (pour revue voir Besse, 1976 ; Maissiat, 1978 ; Meusy, 1980 ; Charniaux-Cotton, 1980 ; Picaud, 1983). Ainsi, selon Besse et Maissiat (1971), le blocage de la mue, provoqué chez Porcellio dilatatus par ablation des glandes de mue (= Y-ectomie), s'accompagne d'un blocage de la vitellogenèse. Ce résultat est confirmé chez Orchestia gammarellus par Meusy et al. (1977) et Blanchet-Tournier (1980) qui montrent que la glande de mue est nécessaire au maintien de la synthèse de la vitellogénine. D'après Andrieux (1981), il semble que chez Carcinus maenas un taux bas d'ecdystéroïdes soit corrélé à la croissance des ovocytes.

L'ablation des glandes de mue, chez l'oniscoïde Porcellio dilatatus, fait chuter la synthèse de la vitellogénine dans le tissu adipeux (Souty et Gohar, en préparation), ainsi que la synthèse et la libération de la vitellogénine dans l'hémolymphe (Souty et al., 1982). Dans le même travail, ces auteurs montrent qu'une injection compensatrice de 20-hydroxyecdysone rétablit cette synthèse et cette libération. 
D'une façon directe ou indirecte, la 20-hydroxyecdysone est nécessaire au maintien de la synthèse de la fraction exogène du vitellus protéique (la vitellogénine) chez Porcellio dilatatus. Cependant, l'action de cette hormone sur la synthèse protéique ovarienne est encore mal connue chez les Crustacés. De plus, si l'on sait que la synthèse de la vitellogénine suit un rythme circadien chez Porcellio dilatatus (Gohar et al., 1983), aucune approche chronobiologique de l'effet des ecdystéroïdes sur la vitellogenèse n'a encore été tentée.

Dans un travail récent, Souty (1983) montre que les femelles en reproduction de Porcellio dilatatus offrent, dans le cycle de mue, une alternance des pics de synthèse de la fraction exogène et des pics de la synthèse protéique ovarienne. Quinze à vingt jours après la mue (Stade $\mathrm{Cb}$ ), la synthèse protéique ovarienne montre un palier. C'est à ce stade que nous avons testé l'action temporelle, dans le nycthémère, de l'ecdysone et de la 20-hydroxyecdysone sur la synthèse protéique ovarienne.

\section{Matériel et méthodes.}

Les femelles de Porcellio dilatatus utilisées proviennent d'élevage en photopériode artificielle de $18 \mathrm{~L} / 6 \mathrm{D}$, et à la température constante de $20^{\circ} \mathrm{C}$. Toutes ces femelles sont gravides et susceptibles d'effectuer une $2^{\mathrm{e}}$ vitellogenèse. Elles se trouvent 15 à 20 jours après la mue, soit en stade $\mathrm{Cb}$ du cycle de mue. Sur chaque animal les deux ovaires sont prélevés et mis $4 \mathrm{~h}$ en culture en salière dans $200 \mu \mathrm{l}$ de milieu. L'incubation est réalisée à $20^{\circ} \mathrm{C}$ en lumière constante et sur un plateau tournant.

Le milieu de culture est constitué de liquide physiologique additionné de $1 \%$ de leucine ${ }^{14} \mathrm{C}$ (Amersham, activité spécifique $350 \mathrm{mCi} / \mathrm{mmole}, 50 \mu \mathrm{Ci} / \mathrm{ml}$ ) et de $5 \%$ de chacun des constituants suivants: jus d'embryons de Poulet (Difco), streptomycine (Specia), specilline (Specia) et polymyxine (Pfizer).

L'un des deux ovaires reçoit $5 \mu$ d'ecdysone (Hoffmann-Laroche) ou de 20-hydroxyecdysone (Rohto Pharmaceutical Cie) en solution dans de l'éthanol à $5 \%$, à la concentration de $1,25 \mu \mathrm{g} / \mu \mathrm{l}$. La concentration finale de l'ecdysone dans le milieu de culture est donc de $31 \mathrm{ng} / \mu \mathrm{l}$. L'ovaire correspondant reçoit $5 \mu \mathrm{l}$ d'éthanol à $5 \%$.

Toutes les quatre heures à partir de 4 h 30 après l'aube, les femelles sont disséquées et leurs ovaires mis en culture. Quatre heures après, les ovaires sont rincés puis homogénéisés par sonication dans du liquide physiologique. Après centrifugation, le surnageant est repris, puis divisé en deux fractions aliquotes.

Sur l'une d'elles, les protéines marquées précipitant avec un immunsérum anti-vitellines (protéines $I s V t$ ) sont dosées. Cet immunsérum est obtenu après injection à un lapin d'un extrait ovarien préparé à partir d'ovaires en fin de vitellogenèse, où la vitellogénine et les vitellines représentent la plus grande partie des protéines ovariennes (Picaud, 1974). La vitellogénine et les vitellines étant des analogues antigéniques (Kerr, 1969 ; Croisille et al., 1974 ; Picaud, 1978), l'immunsérum réagira surtout - sinon exclusivement - contre ces protéines. Le sérum obtenu est épuisé par de l'hémolymphe de mâle (voir Picaud et Souty, 1981 ; Gohar et al., 1983). Sur l'autre fraction, les protéines marquées sont 
dosées après précipitation par l'acide trichloracétique à $20 \%$. Les résultats intermédiaires sont exprimés en coups par minute par ovaire (c.p.m./ovaire). Les méthodes de dosage utilisées sont expliquées plus en détail par Picaud et Souty (1981), Gohar et al. (1983) et Souty (1983). Le résultat final est exprimé sous forme de pourcentage de stimulation $S=\frac{T-C}{C} \times 100$, avec $T=c . p . m . /$ ovaire traité, $\mathrm{C}=$ c.p.m. /ovaire témoin.

\section{Résultats.}

Les résultats obtenus sont rassemblés dans les figures 1 et 2 . L'ecdysone (fig. 1) montre un rythme bimodal de stimulation de l'endosynthèse. L'un des maximums de stimulation est situé le matin, $4 \mathrm{~h}$ après l'aube, l'autre est situé dans la nuit. En fin de journée, l'effet de l'ecdysone sur la synthèse ovarienne devient légèrement inhibiteur. Les protéines totales (fig. 1a) et les protéines IsVt (fig. 1b) offrent une réponse similaire. La synthèse des protéines totales paraît cependant davantage stimulée par l'ecdysone que la synthèse des protéines IsVt. La variation de l'effet de l'ecdysone sur la synthèse des protéines totales au cours du cycle nycthéméral est significative au niveau $1 \%$, celle des protéines IsVt au niveau $5 \%$.
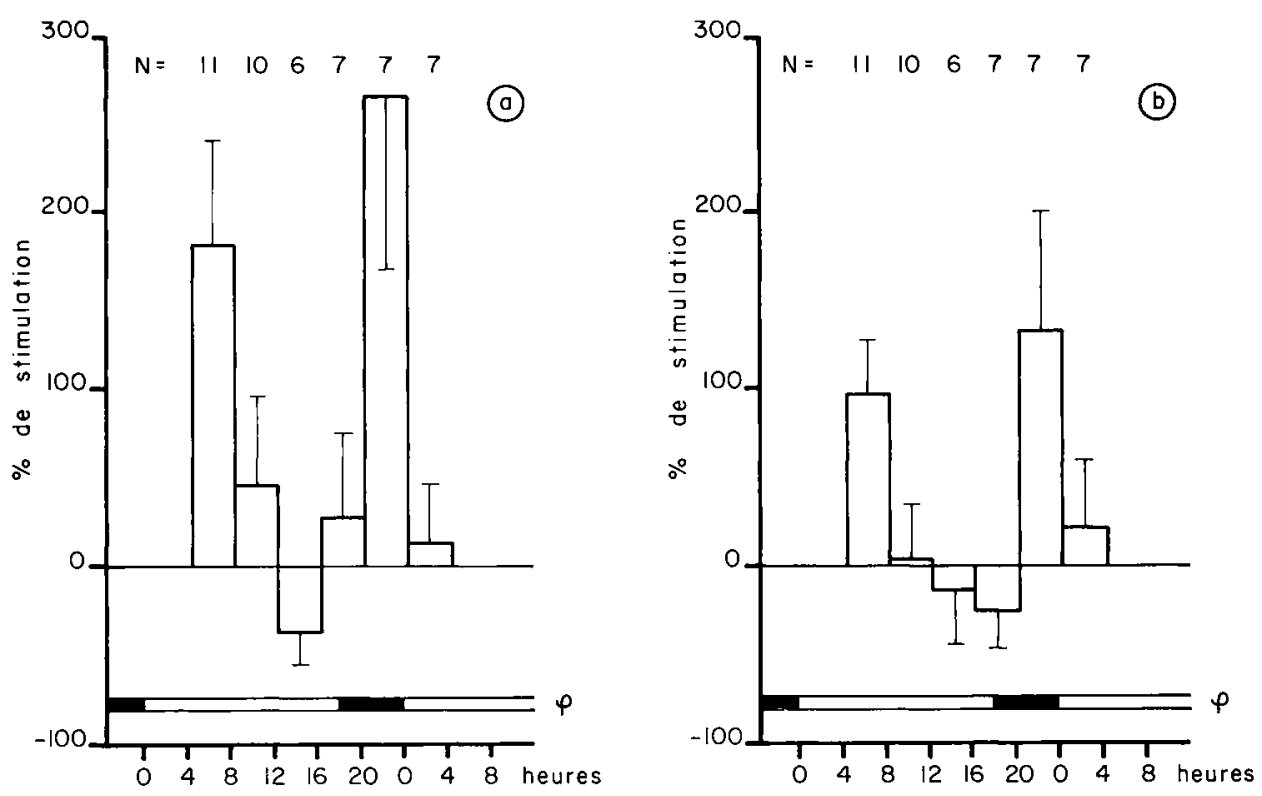

FIG. 1. -- Action temporelle de l'ecdysone sur la synthèse protéique ovarienne.

(a) : Protéines totales; (b) : Protéines Is Vt (Protéines précipitées par l'immunsérum anti-vitellines). $N$ : Echantillon ; $\varphi$ : Photopériode. 
La synthèse protéique ovarienne répond de façon unimodale à l'action temporelle de la 20-hydroxyecdysone au cours du cycle de $24 \mathrm{~h}$ (fig. 2). Le maximum est situé au milieu de la journée, $8 \mathrm{~h}$ après l'aube. Le minimum est placé à la fin du jour et correspond à une faible inhibition. L'amplitude des valeurs obtenues pour la synthèse des protéines totales (fig. 2a) est supérieure à celle constatée dans le cas des protéines Is Vt (fig. $2 \mathrm{~b}$ ). La variation de la réponse de lấ synthèse protéique ovarienne à l'action de la 20-hydroxyecdysone est significative au niveau $5 \%$ tant pour la synthèse des protéines totales que pour la synthèse des protéines IsVt.

\section{Discussion.}

Si l'on excepte le fait que l'amplitude des valeurs obtenues est plus grande pour les protéines totales que pour les protéines $\mathrm{IsVt}$, les données apportées par les deux méthodes de dosage sont identiques. Chez la plupart des Crustacés, I'ovaire synthétise des protéines vitellines (Hinsh et Cone, 1969 ; Lui et al., 1974 ; Lui et O'Connor, 1977 ; Arcier et Bonami, 1979). Chez les Isopodes (Souty, 1980 ; Souty-Grosset, en préparation) comme chez de nombreux Crustacés (Zerbib, 1976, 1980 ; Charniaux-Cotton, 1980 ; De Leernsyder et al., 1980 ; Arnaud et al., 1982), ces protéines vitellines endogènes sont surtout synthétisées au début de la vitellogenèse, tandis que l'incorporation de vitellogénine est importante en fin de vitellogenèse. Puisque les ovaires sur lesquels nous avons effectué nos tests
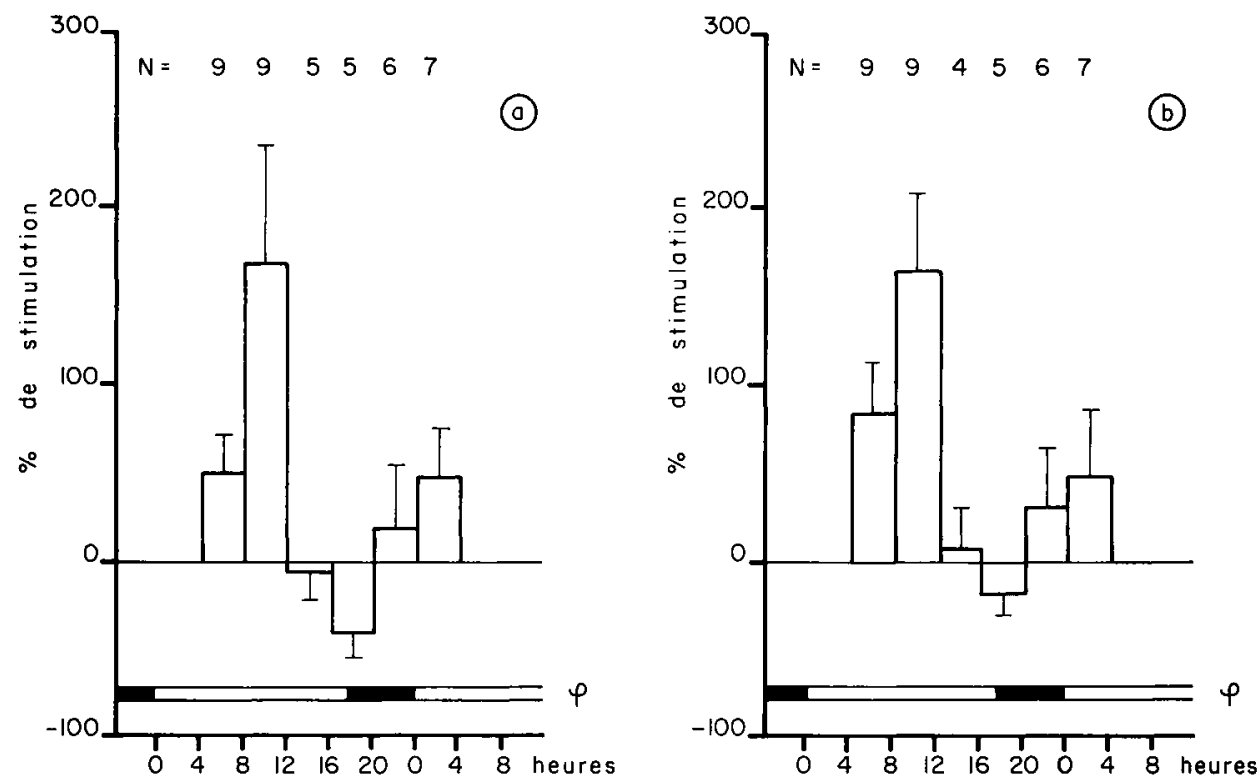

FIG. 2. - Action temporelle de la 20-hydroxyecdysone sur la synthèse protéique ovarienne. (a): Protéines totales; (b): Protéines IsVt.

Autres symboles et conventions voir la figure 1 . 
étaient en début de vitellogenèse, les résultats que nous rapportons ici - notamment pour les protéines Is Vt - concernent très probablement les protéines vitellines synthétisées par l'ovaire. D'autre part, Bekri (1983) détermine, chez les femelles en vitellogenèse et en prémue de Porcellio dilatatus, un maximum de $13 \mathrm{ng} / \mu \mathrm{l}$ d'hémolymphe pour l'ecdysone et de $10 \mathrm{ng} / \mu \mathrm{l}$ d'hémolymphe pour la 20-hydroxyecdysone. Au stade $\mathrm{Cb}$ du cycle de mue, ces concentrations sont, pour l'ecdysone et pour la 20-hydroxyecdysone, respectivement de $5 \mathrm{ng}$ et de 9,4 ng par $\mu \mathrm{l}$ d'hémolymphe.

Par comparaison, rappelons que chez Carcinus maenas Andrieux et al. (1976) déterminent un maximum de $15 \mathrm{ng}$ d'ecdystéroïdes/ $\mu$ d'hémolymphe et que chez Helleria brevicornis Hoarau et Hirn (1978) montrent un maximum de $3 \mathrm{ng}$ d'ecdystéroïdes/ $\mu$ l d'hémolymphe chez les femelles. Par conséquent, bien qu'extra-physiologiques, les concentrations d'ecdystéroïdes adoptées dans ce travail ne s'éloignent pas trop des concentrations maximales observées chez Porce/lio dilatatus et certains autres crustacés.

Ces remarques sur la signification de nos résultats étant faites, nous pouvons classer les problèmes soulevés par ceux-ci dans trois catégories: l'action des ecdystéroïdes sur la synthèse protéique ovarienne, le rythme d'action de ces stéroïdes, enfin la différence entre les rythmes d'action de l'ecdysone et de la 20-hydroxyecdysone.

Effet de l'ecdysone et de la 20-hydroxyecdysone sur la synthèse protéique ovarienne. - Les deux ecdystéroïdes que nous avons utilisés stimulent la synthèse protéique ovarienne et, à des moments particuliers du nycthémère, cette stimulation est forte. Ce résultat est à mettre en parallèle avec l'effet stimulateur de la 20-hydroxyecdysone sur la synthèse de la fraction protéique exogène chez Porcellio dilatatus (Souty et al., 1982 ; Souty et Gohar, en préparation). Cependant, à certains moments de la journée, l'action de ces hormones devient légèrement inhibitrice sur les ovaires. Fyhn et al. (1977) montrent également, chez deux Cirripèdes, une action inhibitrice de la 20-hydroxyecdysone sur la vitellogenèse. Une telle action est décrite par Blanchet et al. (1975) chez la cręvette Lysmata seticaudata. L'utilisation de doses largement extra-physiologiques peut dans certains cas expliquer les effets inhibiteurs des ecdystéroïdes sur la vitellogenèse observés par différents auteurs. Chez Orchestia gammare/lus, Blanchet-Tournier (1982) remarque qu'un taux bas d'ecdystéroïdes est nécessaire à la vitellogenèse. Selon leur concentration et selon le moment de la journée, les ecdystéroïdes seraient inhibiteurs ou stimulateurs de la synthèse protéique ovarienne.

Rythme nycthéméral de l'action de l'ecdysone et de la 20-hydroxyecdysone sur la synthèse protéique ovarienne. - L'effet de l'ecdysone et de la 20-hydroxyecdysone sur la synthèse protéique ovarienne varie dans le cycle de $24 \mathrm{~h}$. De telles variations dans les effets des ecdystéroïdes ont été rapportées pour les phénomènes de mue des Insectes par Truman (1972) chez Antherea permyi, par Truman et al. (1974) chez Manduca sexta et par Fujishita et Ishizaki (1982) et Fujishita et al. (1982) chez Samia cynthia ricini, au cours du développement. Dans nos expérimentations in vitro et sur 4 heures, les variations peuvent être dues à des rythmes dans le taux hémolymphatique des ecdystéroïdes (dont le tissu est impré-

\footnotetext{
Reproduction, Nutrition, Développement, $n^{\circ} 2-1984 .-4$.
} 
gné au moment du prélèvement), à des rythmes dans le catabolisme des ecdystéroïdes ou à des rythmes dans l'apparition des récepteurs ovariens aux ecdystérö̈des. Actuellement, notre connaissance du contrôle de la synthèse des ecdystéroïdes et de leur catabolisme, ainsi que de leur mode d'action sur la synthèse protéique est très incomplète. Cependant, chez les Insectes, d'après Truman (1972) et Fujishita et Ishizaki (1981; 1982), un rythme de neurosécrétion de l'hormone photoracotrope (PTTH) serait responsable de la sécrétion rythmique d'ecdysone par la glande prothoracique. Cette glande serait elle-même, selon Mizoguchi et Ishizaki (1982), un pacemaker. Ces variations seraient donc la résultante de phénomènes complexes, au niveau neuroendocrine, au niveau endocrine et au niveau de l'organe-cible.

Différence entre les rythmes nycthéméraux d'action de l'ecdysone et de la 20-hydroxyecdysone sur la synthèse protéique ovarienne. - La différence entre les actions de l'ecdysone et de la 20-hydroxyecdysone testées sur l'ovaire à des moments particuliers de la journée, semble être d'ordre quantitatif. Cependant, à l'échelle de la journée, cette différence apparaît être principalement d'ordre temporel. Ce résultat est nouveau, seules des différences quantitatives entre les actions de ces deux hormones ayant été observées jusqu'à présent. Ainsi Laverdure (1976) observe in vitro une différence quantitative entre l'action de l'ecdysone et celle de la 20-hydroxyecdysone sur les ovaires en prévitellogenèse de Tenebrio molitor. Les résultats que nous avons obtenus suggèrent que l'ecdysone et la 20-hydroxyecdysone régulent des phénomènes différents impliqués de façon directe ou indirecte dans la synthèse protéique ovarienne. En effet, une activation de l'ecdysone après 20-hydroxylation ne peut pas expliquer ces résultats, puisque au maximum nocture de stimulation de la synthèse protéique ovarienne par l'ecdysone ne correspond qu'une action faible de la 20-hydroxyecdysone.

\section{Conclusion.}

Le rôle des ecdystéroïdes dans le contrôle de la vitellogenèse chez les crustacés est encore mal connu, particulièrement en ce qui concerne la synthèse protéique ovarienne. Les différences que nous avons observées entre l'ecdysone et la 20-hydroxyecdysone dans la stimulation de cette synthèse nous permettent de suggérer que ces hormones ont des actions différentes sur l'ovaire. Ces observations rejoignent celles de Quennedey et al. (1983), qui montrent des effets distincts de l'ecdysone et la 20-hydroxyecdysone sur la différentiation in vitro du tégument d'une jeune pupe de Tenebrio molitor. De même, Spindler et al. (1980) et Kuppert et Spindler (1982) rapportent chez l'Ecrevisse l'existence de récepteurs différents pour l'ecdysone et la 20-hydroxyecdysone.

Malgré l'abondante littérature traitant des rythmes nycthéméraux, les mécanismes à l'origine de ces rythmes sont inconnus. Cependant, comme le souligne Ceccaldi (1981), une dimension temporelle introduite dans les études relatives au contrôle endocrine et neuroendocrine de la reproduction peut constituer une voie de recherche fructueuse. 
Remerciements. - Nous remercions Mme Nasreddine L. pour la dactylographie ainsi que $M$. Bauvais $T$. pour la photographie.

\section{Références}

ANDRIEUX N., 1981. Variations quantitatives et qualitatives des ecdystéroïdes chez Carcinus maenas : étude comparative préliminaire chez les femelles et les individus parasités par Sacculina carcini. C. R. Acad. Sci. Paris, Sér. III, 293, 457-460.

ANDRIEUX N., PORCHERON P., BERREUR-BONNEFANT J., DRAY F., 1976. Détermination du taux d'ecdysone au cours du cycle d'intermue chez le crabe Carcinus maenas ; comparaison entre individus sains et parasités par Sacculina carcini. C. R. Acad. Sci. Paris, Sér. D, 283, 1429-1432.

ARCIER J. M., BONAMI J. R., 1979. Contribution à l'étude des lipovitellines chez Palaemon adspersus (Rathke, 1837). Arch. int. Physiol. Biochim., 87, 471-484.

ARNAUD J., BRUNET M., MAZZA J., 1982. Etude de l'ovogenèse chez Centropages typicus (Copepoda, Calanoida). Reprod. Nutr. Dévelop., 22, 537-555.

BEKRI A., 1983. Contribution à l'étude des dosages des ecdystéroïdes chez le Crustacé lsopode Porcellio dilatatus Brandt. Th. Doct. $3^{e}$ cycle, Univ. Poitiers.

BESSE G., 1976. Contribution à l'étude expérimentale de la physiologie sexuelle femelle chez les Crustacés /sopodes terrestres. Th. Doct. Etat, Univ. Poitiers, CNRS, France $n^{\circ}$ AO, 13017.

BESSE G., MAISSIAT J., 1971. Action de la glande de mue sur la vitellogenèse du Crustacé Isopode Porcellio dilatatus (Brandt). C. R. Acad. Sci., Paris, Sér. D, 273, 1975-1978.

BLANCHET-TOURNIER M. F., 1980. Etude du contrôle endocrine du démarrage de la vitellogenèse secondaire chez le Crustacé Amphipode Orchestia gammarella (Pallas). C. R. Acad. Sci. Paris, Sér. D, 272, 307-310.

BLANCHET-TOURNIER M. F., 1982. Quelques aspects des interactions hormonales entre la mue et la vitellogenèse chez le Crustacé Amphipode Orchestia gammarellus (Pallas). Reprod. Nutr. Dévelop., 22, 325-344.

BLANCHET M. F., JUNERA H., MEUSY J. J., 1975. Mue et vitellogenèse chez Orchestia gammarella Pallas (Crustacé Amphipode) : étude de la synthèse de la fraction protéique femelle après introduction d'ecdystérone. Expérientia, 31, 865-867.

CECCALDI H. J., 1981. Problèmes posés par les variations chronobiologiques de la physiologie des crustacés. Bull. Soc. Ecophysiol., 6, 87-95.

CHARNIAUX-COTTON H., 1980. Experimental studies of reproduction in Malacostraca Crustaceans. Description of vitellogenesis and of its endocrine control, 177-186. In W. H. CLARK Jr, T. S. ADAMS, Advances in invertebrate reproduction, Elsevier North Holiand.

CROISILLE Y., JUNERA H., MEUSY J. J., CHARNIAUX-COTTON H., 1974. The female specific protein (vitellogenic protein) in Crustacea with particular reference to Orchestia gammarellus (Amphipoda). Amer. Zool., 14, 1219-1228.

DE LEERNSYDER M., DHAINAUT A., PORCHERON P., 1980. La vitellogenèse chez le crabe Eriocheir sinensis. Bull. Soc. Zool. Fr., 105, 413-419.

FUCHS M., KANG S. H., 1981. Ecdysone and mosquito vitellogenesis : a critical appraisal. Insect Biochem., 11, 627-633.

FUJISHITA M., ISHIZAKI H., 1981. Circadian clock and prothoracicotropic hormone secretion in relation to the larval-larval ecdysis rhythm of the saturniid Samia cynthia ricini. J. Insect Physiol., 27, 121-128.

FUJISHITA M.,ISHIZAKI H., 1982. Temporal organization en endocrine events in relation to the circadian clock during larval-larval development in Samia cynthia ricini. J. Insect Physiol., 28, 77-84.

FUJISHITA M., OHNISHI E., ESHIZAKI H., 1982. The role of ecdysteroids in the determination of gut-purge timing in the saturniid, Samia cynthia ricini. J. Insect Physiol., 28, 961-967.

FYHN O. F., FYHN H. J., COSTLOW J. D., 1977. Cirriped vitellogenesis : effect of ecdysterone in vitro. Gen. comp. Endocrinol., 32, 266-271. 
GOHAR M., SOUTY C., PICAUD J. L., 1983. Diurnal rhythms in the synthesis and release of haemolymph proteins in the crustacean Isopoda, Porcellio dilatatus, with special reference to vitellogenin. Reprod. Nutr. Develop., 23, 93-100.

HANDLER A. M., 1982. Ecdysteroid Titers during pupal and adult development in Drosophila melanogaster. Develop. Biol., 93, 73-82.

HINSH G. W., CONE M. V., 1969. Ultrastructural observations of vitellogenesis in the spider crab Libinia emarginata. J. Cell Biol., 40, 336-342.

HOARAU F., HIRN M., 1978. Evolution du taux des ecdystéroïdes au cours du cycle de mue chez Helleria brevicornis Ebner (Isopode terrestre). C. R. Acad. Sci. Paris, Sér. D, 286, 1443-1446.

HOFFMANN J., 1980. Ecdysone et reproduction chez les femelles adultes d'insectes. Reprod. Nutr. Dévelop., 20, 443-456.

hOFFMANN J. A., LAgUeUX M., HeTRU C., CHARLET M., GOLTZENE F., 1980. Ecdysone in reproductively competent female adults and in embryos of insects. In J. A. HOFFMANN, Progress in ecdysone, Elsevier North Holland Biomed. Press.

KERR M. S., 1969. The hemolymph proteins of the blue crab, Callinectes sapidus. II. A lipoprotein serologically identical to oocyte lipovitellin. Develop. Biol., 20, 1-17.

KUPPERT P. G., SPINDLER K. D., 1982. Characterization of nuclear ecdysteroid receptor from crayfish integument. J. Steroid Biochem., 17, 205-210.

LAVERDURE A. M., 1976. Action de l' $\alpha$ - et de la $\beta$-ecdysone sur l'évolution de l'ovaire de Tenebrio molitor. Etude en culture in vitro. Coll. Int. CNRS, 251, 367-373.

LUI C. W., O'CONNOR J. D., 1977. Biosynthesis of lipovitellin. III. The incorporation of labeled amino acids into the purified lipovitellin of the crab. Pachygrapsus crassipes. J. exp. Zool., 199, 105-108.

LUI C. W., SAGE B. A., O'CONNOR J. D., 1974. Biosynthesis of lipovitellin by the crustacean ovary. J. exp. Zool., 188, 289-296.

MIZOGUCHI A., ISHIZAKI H., 1982. Prothoracic glands of the saturniid moth Samia cynthia ricini possess a circadian clock controlling gut purge timing. Proc. nat. Acad. Sci., USA, 79, 27262730.

MAISSIAT J., 1978. Contribution à l'étude de la mue et du rôle de l'hormone de mue dans divers processus physiologiques: vitellogenèse, régénération chez les Crustacés Isopodes. Th. Doct. Etat, Univ. Poitiers.

MEUSY J. J., 1980. Vitellogenin, the extraovarien precursor of the protein yolk in Crustacea : a review. Reprod. Nutr. Dévelop., 20, 1-21.

MEUSY J. J., BLANCHET M. F., JUNERA H., 1977. Mue et vitellogenèse chez le Crustacé Amphipode Orchestia gammarella Pallas. II. Etude de la synthèse de la vitellogénine (" Fraction protéique femelle" de l'hémolymphe) après destruction des organes Y. Gen. comp. Endocrinol., 33, 35-40.

PICAUD J. L., 1974. Contribution à l'étude par électrophorèse des protéines des Oniscoïdes. Th. Doct. $3^{\mathrm{e}}$ cycle, Univ. Poitiers.

PICAUD J. L., 1978. Parentés antigéniques des protéines spécifiques femelles chez quelques Crustacés Isopodes. C. R. Soc. Biol., 172, 320-324.

PICAUD J. L., 1983. Les protéines spécifiques femelles des Crustacés Isopodes. Constitution, synthèse et certains aspects du contrôle de leur synthèse et de leur libération. Th. Doct. Etat, Univ. Poitiers.

PICAUD J. L., SOUTY C., 1981. Approche quantitative de I'influence de l'ovariectomie sur la synthèse de la vitellogénine chez Porcellio dilatatus Brandt (Crustacé, Isopode). C. R. Acad. Sci., Paris, Sér. III, 293, 479-482.

QUENNEDEY A., QUENNEDEY B., DELBECQUE J. P., DELACHAMBRE J., 1983. The in vitro development of pupal integument and the effects of ecdysteroids in Tenebrio molitor (Insecta, Coleoptera). Cell. Tissue Res., 232, 493-511.

SPINDLER K. D., KELLER R., O'CONNOR J. D., 1980. The role of ecdysteroids in the Crustacean molting cycle, 247-280. In J. A. HOFFMANN, Progress in ecdysone research, Elsevier/NorthHolland. Biomed. Press.

SOUTY C., 1980. Electron microscopy study of follicle cell development during vitellogenesis in the marine Crustacean Isopoda, Idotea balthica basteri. Reprod. Nutr. Dévelop., 20, 653-663. 
SOUTY C., 1983. Démonstration d'une alternance des pics de synthèse des fractions, respectivement endogène et exogène, du vitellus protéique chez l'Oniscoïde Porcellio dilatatus Brandt. C. R. Acad. Sci. Paris. Sér. III, 294, 1057-1059.

SOUTY C., BESSE G., PICAUD J. L., 1982. Stimulation par l'ecdysone du taux hémolymphatique de la vitellogénine chez le Crustacé Isopode terrestre Porcellio dilatatus Brandt. C. R. Acad. Sci. Paris, Sér. D, 294, 1057-1059.

TRUMAN J. W., 1972. Physiology of insect rhythms. I. Circadian organization of the endocrine events underlying the moulting cycle of larval tobacco hornworms. J. exp. Biol., 57, 805-820.

TRUMAN J. W., RIDDIFORD L. M., SAFRANEK L., 1974. Temporal patterns of response to ecdysone and juvenile hormone in the epidermis of the tobacco hornwornm, Manduca sexta. Develop. Biol., 39, 247-262.

ZERBIB C., 1976. Nature chimique des enclaves vitellines de l'ovocyte du Crustacé Amphipode Orchestia gammarellus (Pallas). Ann. Histochim., 21, 279-295.

ZERBIB C., 1980. Ultrastructural observation of oogenesis in the Crustacea Amphipoda Orchestia gammarellus (Pallas). Tissue Cell, 12, 47-62. 